\title{
Transition to schizophrenia in acute and transient psychotic disorders
}

Filippo Queirazza, David M. Semple and Stephen M. Lawrie

\section{Background}

The diagnostic category of acute and transient psychotic disorders (ATPD) was introduced in ICD-10. Subsequent studies have called into question its validity and reliability.

\section{Aims}

To determine the pattern of diagnostic revision to schizophrenia in first-ever diagnosed ATPD.

\section{Method}

Using data drawn from the Scottish Morbidity Record, we estimated incidence and diagnostic change in first-ever diagnosed ATPD in Scottish hospitals between January 1997 and December $2010(n=2923)$

\section{Results}

The average incidence of ATPD was 4.1 per 100000 population per year. Diagnostic stability was estimated at $53.9 \%$ over an average of approximately 4 years of observation. The most common diagnostic shift was to schizophrenia (12.6\%), over an average of 1.7 years Estimates of the transition risks for schizophrenia were $80 \%$ at 2.8 years and $90 \%$ at 4.6 years. Longer first admission to hospital, younger age at onset and male gender were associated with increased risk and earlier development of schizophrenia.

\section{Conclusions}

Routinely collected data suggest that approximately one in eight individuals with first-ever diagnosed ATPD will develop schizophrenia within 3-5 years. Those at high risk of transition may benefit from monitoring for possible diagnostic change.

\section{Declaration of interest}

None.
The Kraepelinian dichotomy of functional psychoses into dementia praecox and manic-depressive insanity never accommodated non-affective acute and rapidly remitting psychotic disorders with a favourable outcome. ${ }^{1,2}$ The concept of a 'third psychosis' has, however, been described across many different psychiatric traditions, ${ }^{3}$ including Kleist and Leonhard's 'cycloid psychoses' in Germany, ${ }^{4,5}$ Magnan and Legrain's 'bouffée délirante' in France, ${ }^{6,7}$ Wimmer's 'psychogenic psychoses' ${ }^{8}$ and Langfeldt's 'schizophreniform psychoses' ' in Scandinavia. In ICD-10 the World Health Organization brought these clinical concepts together into the diagnostic category of acute and transient psychotic disorders (ATPD) but also acknowledged the uncertainty of their nosological status. ${ }^{10}$ Comparative studies have revealed ATPD has little continuity with its apparent historical predecessors. ${ }^{11,12}$ Acute and transient psychotic disorders is a composite category encompassing six subtypes, in which symptom resolution is expected to occur within 1 or 3 months in order to exclude schizophrenia and persistent delusional disorder respectively. Furthermore, premorbid adjustment and long-term functional outcome have been demonstrated to be fairly similar to those of the general population and better than those observed in schizophrenia. ${ }^{13-15}$ One method of determining the validity of the ATPD diagnostic concept is to test its predictive power. ${ }^{16,17}$ Follow-up studies to establish the prognostic validity, longitudinal course and long-term outcome of ATPD have suggested a low to moderate diagnostic stability of ATPD, with generally higher transition rates to other diagnoses in studies conducted in Europe and Japan ${ }^{18-22}$ than in low- and middle-income countries. ${ }^{23-27}$ In this study we analysed readmission patterns of all individuals listed in the Scottish Morbidity Record 4 (SMR4) (http:// isdscotland.org/Health-Topics/Mental-Health/Psychiatric-HospitalActivity/) as having been first-ever admitted to a psychiatric hospital with a diagnosis of ATPD from 1 January 1997 until 31 December 2010. Our first aim was to establish the transition rate and mean time to shift to schizophrenia of individuals with new-diagnosis
ATPD in a population of psychiatric in-patients across Scotland. To the best of our knowledge, no previous study has ever estimated mean time to change in diagnosis from ATPD to schizophrenia. This knowledge could potentially contribute to better planning and design of first-episode psychosis services in the UK. Our second objective was to establish whether any demographic (gender and age at first presentation) or clinical (length of first admission) variables were significantly associated with a subsequent diagnosis of schizophrenia. As a corollary, we sought to determine the incidence of new-diagnosis ATPD, its diagnostic stability and the mean time to diagnostic revision from ATPD to any other diagnosis.

\section{Method}

\section{Sample characteristics and inclusion criteria}

The Information Services Division (ISD) is a division of National Services Scotland, part of NHS (National Health Service) Scotland. A primary role of ISD is to collate, analyse, interpret and publish information about health and healthcare in Scotland for and on behalf of the Scottish Government and NHS Scotland. Mental health activity data are collected across NHS Scotland and are based on nationally available information routinely drawn from hospital administrative systems across the country. The principal data source is the SMR4 return, which collects information on admissions to and discharges from NHS mental health hospitals and psychiatric in-patient units within general hospitals in Scotland. The information stored in the SMR4 database can be requested via ISD's Information Request Service and is not part of the NHS Scotland patient management systems, which give healthcare staff across Scotland immediate access to healthcare information. Anonymisation of the national databases and adherence to a Statistical Disclosure Control Protocol based on the guidance released by the UK Office of National Statistics 
ensure protection of any personally identifiable information. Admissions to psychiatric hospitals in Scotland are classified into three main types (as recorded by the psychiatric hospital when a patient is admitted): 'first admissions' (patients who have not previously received psychiatric in-patient care); 'readmissions' (patients who are readmitted following a break from psychiatric in-patient care); 'transfers' (direct transfer from another psychiatric hospital or from one consultant to another within the same hospital). In order to obtain an incidence cohort, we initially selected only 'first admissions' and then retrieved all the subsequent admissions to hospital over the follow-up period. The use of ICD-10 diagnostic codes was first introduced in the SMR4 data-set in 1997. We selected all those with a main diagnosis of ICD-10 code F23 - acute and transient psychotic disorders - admitted between 1 January 1997 and 31 December 2010. Diagnosis was selected for diagnosis on discharge. This is understood to be a more robust and complete method of measuring diagnosis as in some cases a specific diagnosis may not be reported and a diagnosis such as 'under observation' may be recorded instead. Age was selected for age at admission as, in some cases, there may have been a significant length of time between admission and discharge. Gender distribution of new diagnoses of ATPD, mean and standard deviation (s.d.) of age at first admission and of length of first admission were tabulated. Independent $t$-test was performed to compare mean incidence rates, mean age at first admission and mean length of first admission in men and women.

\section{Transition rate and mean time to change to schizophrenia}

The proportion of patients with a change in diagnosis to schizophrenia was calculated. Kaplan-Meier survival analysis with censoring was performed to examine the survival time of the entire data-set from a first-recorded diagnosis of ATPD to specific diagnosis of schizophrenia (F20.0) and to any new diagnosis other than recurrent ATPD. For diagnosis of schizophrenia, separate groups of patients defined by gender, length of first admission ( $>14$ days) and age at first admission ( $\geqslant 30$ years) were compared using plots of cumulative survival by period of observation. Tests of homogeneity of survival across these strata were conducted using the log-rank test and, where significant, were entered into a Cox proportional hazards analysis. Plots of cumulative survival, one minus cumulative survival, log survival, $\log$ minus log survival and cumulative hazard were obtained for illustration and to check model assumptions. Interactions were also explored to see whether there was any interdependence between the significant variables identified.

\section{Demographic and clinical variables associated with diagnostic change to schizophrenia}

The data-set was stratified into four groups: (a) transition to other diagnosis (not F20 or recurrent F23); (b) transition to F20; (c) recurrent F23 episodes; and (d) single F23 episode. Summaries of gender, age at first admission and length of first admission were tabulated. Between-group comparisons were performed using factorial ANOVA for mean age at first admission and mean length of first admission. Chi-squared tests were performed to compare the percentages of men and women across the groups.

\section{Incidence and diagnostic stability}

The annual incidence rate of ATPD was calculated using Scottish Office statistics for the Scottish population (www.gro-scotland. gov.uk/statistics/theme/population/estimates/mid-year/index.html). Each individual patient was identified through a unique patient number so that we could then retrieve all subsequent admissions to hospital over the follow-up period and check for any diagnostic shift. For patients with different diagnoses at subsequent admissions, the most consistent diagnosis was regarded as the diagnostic transition and the time to the earliest formulation of that diagnosis as the time to change in diagnosis. Incidence of new-diagnosis ATPD sorted by various age groups $(<20,20-29$, $30-39,40-49,50-59,60-69,70-79,>79$ ) was plotted against gender. The proportions of patients with a change in diagnosis were calculated. All changes in diagnosis as well as mono-episodic ATPD were sorted by the same age groups.

We used SPSS (statistics release 17.0.0 for Windows) to perform data analysis. Significance was assumed with $P<0.05$.

\section{Results}

\section{Sample demographics}

We identified 2923 cases of first-admission ATPD (1593 men and 1330 women) and retrieved a total number of 8265 subsequent hospital admissions. Male in-patients represented $54.5 \%$ of our sample. The mean age at first admission was 37.4 years (s.d. = 17.7) and the mean length of first admission was 34.8 days (s.d. $=138.5)($ Table 1$)$.

\section{Transition rate and mean time to change to schizophrenia}

In our sample, schizophrenia was the most common diagnostic revision (12.6\%), meaning that one in eight of individuals with a first-ever diagnosed ATPD were later reclassified as having schizophrenia (Fig. 1). The mean length of follow-up was 4 years (s.d. $=3.97$, median 2.49, range 0-14.05). Survival analysis with censoring was performed and the mean time to change from new-diagnosis ATPD to any diagnosis other than recurrent ATPD was 7.57 (95\% CI $7.32-7.82$, s.e. $=0.12)$. For the 369 patients who received a diagnosis of schizophrenia, mean time to diagnosis was 1.7 years (95\% CI 1.5-1.9) (Fig. 2). Estimates of the transition risks in this subgroup of patients were $80 \%$ at 2.8 years and $90 \%$ at 4.6 years. Comparing outcomes for men and women using the log-rank test revealed a significant difference in the mean time to change to a diagnosis of schizophrenia $\left(\chi^{2}=32.76\right.$, d.f. $=1$, $P<0.001)$ between men $(1.48$ years, s.e. $=0.11)$ and women

\begin{tabular}{|c|c|c|c|c|c|}
\hline & \multirow[b]{2}{*}{$n(\%)$} & \multicolumn{2}{|c|}{ Age at first admission, years } & \multicolumn{2}{|c|}{ Length of first admission for F23, days } \\
\hline & & Mean (s.d.) & $P$ & Mean (s.d.) & $P$ \\
\hline Men & $1593(54.5)$ & 33.8 (15.4) & $<0.001$ & $31.7(55.6)$ & 0.19 \\
\hline Women & $1330(45.5)$ & 41.8 (19.3) & $<0.001$ & $38.5(196.1)$ & 0.19 \\
\hline Total & $2923(100)$ & $37.4(17.7)$ & & 34.8 (138.5) & \\
\hline
\end{tabular}




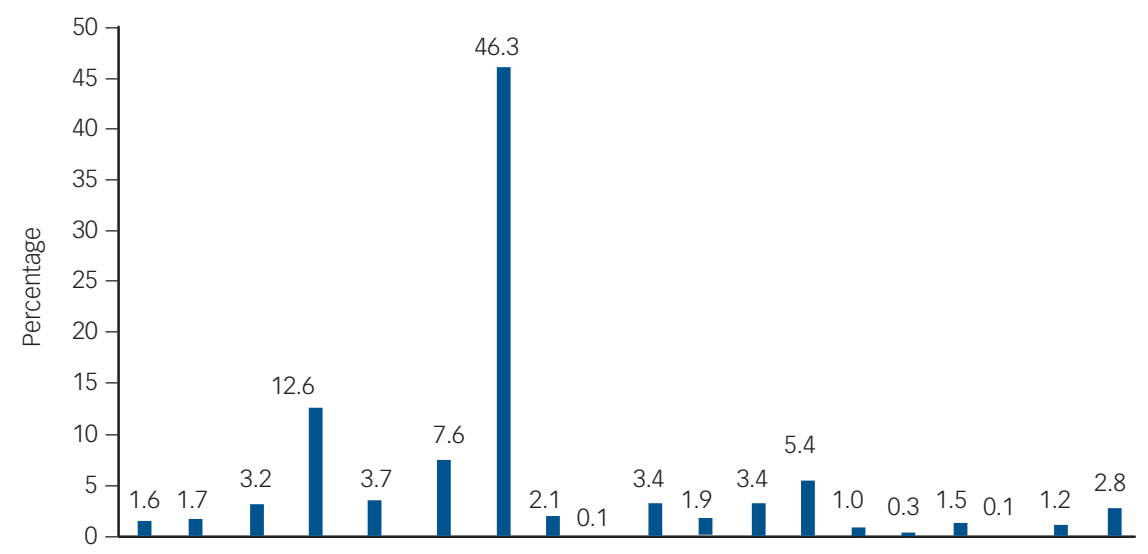

F0X F10 F1X F20 F22 F23R F23X F25 F28 F29 F30 F31 F32 F33 F3X F4X F5X F6X Other

ICD-10 diagnostic code

Fig. 1 Percentages of ICD-10-coded diagnostic changes in a cohort of patients first-ever admitted to any Scottish psychiatric hospital between 1997 and 2010 with a main diagnosis of acute and transient psychotic disorders.

F0X, dementias; F10, mental and behavioural disorders due to alcohol; F1X, mental and behavioural disorders due to other psychoactive substances; F20, schizophrenia; F22, persistent delusional disorder; F23R, recurrent ATPD episodes; F23X, single acute and transient psychotic disorder episode; F25, schizoaffective disorder; F28, other non-organic psychotic disorder; F29, unspecified non-organic psychosis; F30, manic episode; F31, bipolar affective disorder; F32, depressive episode; F33, recurrent depressive disorder; F3X, persistent, other and unspecified mood disorders; F4X, neurotic, stress-related and somatoform disorders; F5x, behavioural syndromes associated with physiological disturbances and physical factors; F6X, disorders of adult personality and behaviour; Other, F7x mental retardation, F8x disorders of psychological development, F9x behavioural and emotional disorders with onset usually occurring in childhood and adolescence.

$(2.15$ years, s.e. $=0.2)$. These data suggest that men convert to schizophrenia sooner than women. Multivariate logistic Cox's proportional hazards analysis found a significant influence of male gender (hazard ratio $(\mathrm{HR})=1.620$, 95\% CI 1.299-2.019), age at first admission under 30 years $(\mathrm{HR}=2.183,95 \% \mathrm{CI}$ 1.767-2.698) and length of first admission over 14 days $(\mathrm{HR}=0.649,95 \% \mathrm{CI} 0.521-0.808)$ on time to change in diagnosis to schizophrenia.

\section{Demographic and clinical variables associated with diagnostic change to schizophrenia}

Between-group comparisons revealed a significantly higher proportion of individuals of younger age (mean 30.4 years, s.d. $=13.6)$ and male gender $(66.7 \%)$ in those who converted to F20 $(n=369)$ than in those who presented with a single F23 episode $(n=1354$, mean 40.1 years, s.d. $=18.9,54.5 \%$ men $)$, recurrent F23 episodes $(n=223$, mean 37.3 , s.d. $=16.0,50.2 \%$ men) or who later received a non-F20 diagnosis $(n=997$, mean 36.4 years, s.d. $=16.9,55.6 \%$ men). Mean length of first admission was shorter for the non-F20 conversion group (28.3 days, s.d. $=42.3)$ and the recurrent F23 group (23.2 days, s.d. $=37.9)$ compared with the single F23 (37.5 days, s.d. $=195.7)$ and F20 (41.2 days, s.d. $=75.1)$ groups but this was not statistically significant. In those who converted to schizophrenia the mean age was significantly higher in women $(35$ years, s.d. $=15.01)$ than in men $(28$ years, s.d. $=12.26)(t=-4.50$, d.f. $=205.62, P<0.001)$.

\section{Incidence and diagnostic stability}

The average incidence of new-diagnosis ATPD was 4.1 (s.d. $=0.47$ ) per 100000 population (range 3.3-4.7). There was very little variation in this incidence over the follow-up period (Fig. 3). The average incidence rate was significantly higher in men than in women ( 4.6 v. 3.5 per 100000 ; $t=4.47$, d.f. $=16.40, P<0.001)$. The mean age at admission was significantly higher in women than men ( $41.8 v$ v. 33.8 years; $t=-12.39$, d.f. $=2921.00, P<0.001)$. The mean length of first admission was longer for women than for men (38.5 v. 31.7 days) but the difference was not statistically significant $(t=-1.32$, d.f. $=921.00, P=0.19)$. Gender distribution of new-diagnosis ATPD by age group revealed higher and earlier peak incidence rates for men than women with a reversal of this pattern over 40 years. Moreover, in both genders higher incidence rates of ATPD were found in the third and fourth decade of life.

Of the ATPD population, $46.3 \%$ were never readmitted and were therefore classified as 'single F23 episode'. Moreover, 7.6\% went on to have a history of recurrent acute and transient psychotic episodes (Fig. 1). Other significant diagnostic shifts are also shown in Fig. 1: persistent delusional disorder (3.7\%), schizoaffective disorder (2.1\%), unspecified non-organic psychosis $(3.4 \%)$, other non-organic psychosis $(0.1 \%)$, personality and

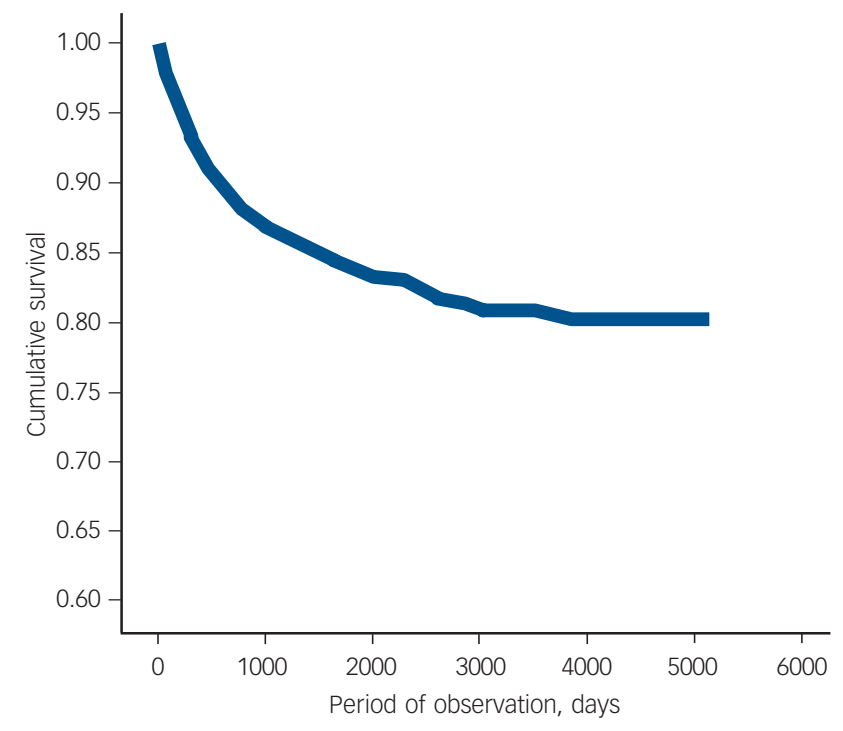

Fig. 2 Survival analysis with censoring illustrating time to change from acute and transient psychotic disorder (ATPD) to schizophrenia in a cohort of patients first-ever admitted to any Scottish psychiatric hospital between 1997 and 2010 with a main diagnosis of ATPD. 


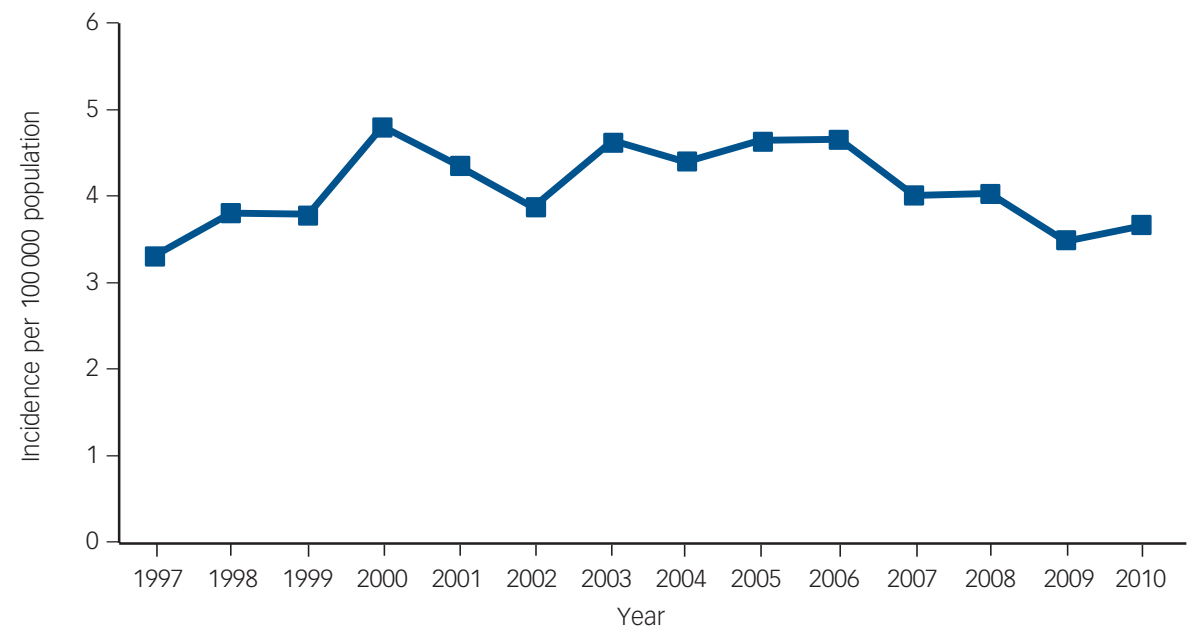

Fig. 3 Incidence of new-diagnosis acute and transient psychotic disorders across Scottish psychiatric hospitals between 1997 and 2010.

behaviour disorders $(1.2 \%)$, anxiety or stress-related disorders $(1.5 \%)$, unipolar depression $(5.4 \%)$, bipolar affective disorder $(3.4 \%)$, recurrent depressive disorder $(1.0 \%)$, mental and behavioural disorders due to alcohol $(1.7 \%)$ and to other psychoactive substances (3.2\%), and organic mental disorders $(1.6 \%)$. Plotting the proportions of diagnostic change and mono-episodic ATPD sorted by various age groups suggested intriguing effects. Increasing rates for mono-episodic ATPD were found in progressively older age groups, ranging from $35.5 \%$ in the under 20 and $62.6 \%$ in the over 69 age groups. Conversion rates to schizophrenia exhibited a completely reverse pattern, peaking at $21.5 \%$ in the under 20 age group, then gradually decreasing to $8.7 \%$ in the seventh decade of life, apart from a sharp fall to $6.3 \%$ in the $40-49$ age group, and then dropping to $2.6 \%$ in the over 69 age group. Rates of recurrent ATPD oscillated between 7 and $8 \%$ in the second, third, fourth and fifth decades of life; peaked at $12.8 \%$ in the sixth decade of life, returned to $8 \%$ in the seventh decade of life and finally dropped to $3.9 \%$ in the over 69 age group.

\section{Discussion}

\section{Transition to schizophrenia}

In our study the most common diagnostic change of first-episode ATPD was to schizophrenia $(12.6 \%)$ and in general to schizophrenia spectrum disorders $(22.3 \%)$. According to our data, one in eight patients with first-episode ATPD will be diagnosed with schizophrenia over an average of 1.7 years. Moreover, one in five of those who developed schizophrenia were younger than 20 years. Our data are consistent with previous studies that found the predominant pattern of diagnostic change from first-episode ATPD was to schizophrenia spectrum disorders. ${ }^{20,21,28,29}$ Interestingly, some earlier reports appear to indicate the type of ATPD subsyndromes may have some bearing on subsequent diagnostic revisions, with the 'polymorphic' subgroups being more likely to transition to affective disorders and the 'non-polymorphic' subgroups being more likely to be later reclassified as schizophrenia. $^{26,28,30}$ It is worth noting that some previous studies reporting high stability rates for ATPD, included samples of patients with acute polymorphic psychotic disorder only. ${ }^{24,26}$ It would therefore appear that the presence of polymorphous (cycloid) features may predict a relatively more stable and more reliable diagnostic construct than a schizophrenia-like clinical picture, which may instead represent a prodromal phase of a schizophrenia spectrum disorder. Although there may be some evidence to the contrary, ${ }^{20}$ this view has been recently supported by data from the McLean-Harvard International First-Episode Project, which found after a 2-year follow-up that $67 \%$ of patients retained their initial diagnosis of acute polymorphic psychotic disorder with and without schizophrenic symptoms (F23.0 and F23.1) and only $28.6 \%$ of patients maintained their initial diagnosis of acute schizophrenia-like psychotic disorder. ${ }^{30}$ Furthermore, Castagnini et al found that ATPD subgroups with schizophrenic symptoms (F23.1 and F23.2) were more prevalent in men and also showed an earlier age at onset than other ATPD subgroups, which the authors suggested may point to a close kinship with schizophrenia of these subgroups. ${ }^{21}$ Based on this evidence, it has been anticipated that in the upcoming revision of ICD-10 the ATPD subgroups with schizophrenic symptoms may be collapsed into the 'other primary psychotic disorders' category and only the pure polymorphous subtype may be retained in the new definition of ATPD. ${ }^{31}$

Of all the recorded diagnostic changes, schizophrenia is the mental disorder with the worst prognosis and poorest long-term global functioning. Thus, the identification of features delineating those whose condition will convert to schizophrenia from the remainder of those with a first-episode ATPD could enable implementation of early-intervention strategies and subsequent amelioration of prognosis in this group of patients. In our sample, analysis of variance of diagnostic subgroup characteristics revealed male gender and younger age at first presentation to be significantly associated with diagnostic transition to schizophrenia. A non-significant trend for longer first admission to hospital in association with later development of schizophrenia was also found. Previous research indicated that poor premorbid social adaptation ${ }^{13}$ and early recurrence of psychosis ${ }^{22}$ may be other useful predictors of future development of schizophrenia. Furthermore, as far as the phenomenology of ATPD is concerned, negative symptoms, ${ }^{14}$ rapidly changing clinical features and anxiety $^{32}$ during the acute psychotic episode have been found to be discriminating factors for schizophrenia and ATPD respectively. Even though the presence of these early predictors should arouse clinical suspicion of later transition to schizophrenia, longitudinal follow-up remains the only reliable method to elucidate diagnosis. For this reason, of particular clinical relevance was the survival analysis regarding this subgroup of patients. Kaplan-Meier estimates of the transition risks were 
$80 \%$ at 2.8 years and $90 \%$ at 4.6 years. The mean time to change to a diagnosis of schizophrenia in our sample was 1.7 years. Men turned out to switch sooner than women ( 1.5 v. 2.2 years). Of particular note, in our sample, is that the chance of earlier development of schizophrenia was $74 \%$ higher when duration of first hospital admission exceeded 14 days, 68\% higher if age at onset was younger than 30 and $62 \%$ higher for male gender. Taken together, the above findings suggest that young age at first presentation, male gender and non-rapidly remitting psychotic symptoms in the context of the emergence of clinical features resembling ATPD may be an early manifestation of an underlying schizophrenic disease process and that close monitoring of patients with first-episode ATPD with such characteristics over a follow-up period of 3 years or more is warranted in order to confidently exclude a diagnosis of schizophrenia.

\section{Incidence}

Our study found an overall incidence rate for patients admitted to Scottish hospitals with first-episode ATPD of 4.1 per 100000 population. The range of the annual incidence rates was between 3.3 and 4.7 per 100000 population. Previously, Singh et $a l^{20}$ found an annual incidence rate of 3.9 per 100000 in the population of Nottingham, and Castagnini et $a l^{21}$ found an annual incidence rate of 9.6 per 100000 in the Danish population using data drawn from the Danish psychiatric central register. Both these previous studies used incidence cohorts, which also included patients treated in out-patient services. It can be argued that because of the lack of data from Scottish out-patient clinics our incidence rate may be a slight underestimation of the overall incidence rate of ATPD in the Scottish population, but our figures are in line with those of Singh et al, and the typically florid and dramatic nature of ATPD psychopathology means that only a minority are likely to be successfully managed in the community without requiring admission to hospital. Hence, our incidence rate is likely to represent an accurate estimate of the incidence rate of ATPD in the Scottish population. The much higher rates in the Danish population of Castagnini et $a l^{21}$ may reflect diagnostic habits regarding the concept of reactive psychosis, previously commonly used in Denmark and then incorporated into the paradigm of ATPD in ICD-10, possibly resulting in an overestimation of its incidence rate. It is also worth noting that the stability of the incidence of new-diagnosis ATPD that we observed over the 13year recruitment period suggests a consistency of diagnostic practice across time in psychiatrists practising in Scotland, which further supports the validity of our results.

\section{Gender}

In our sample we found a slight male preponderance with a male/ female ratio of 1.19. This compares with a reduced prevalence of ATPD in women reported by Castagnini et al with a male/female ratio of $0.93 .^{21}$ Singh et al instead found a marked male preponderance with a male/female ratio of $1.87 .^{20}$ This latter finding appeared to be accounted for by a higher rate of false positive diagnosis of ATPD in men then in women. In our sample too, men were less likely to retain their initial diagnosis than women, so that if the 'true' incidence rate of ATPD was to be considered there would be a female preponderance. Indeed, even though so far clinical consensus has been that ATPD mainly affects females, ${ }^{1}$ a number of previous studies showing female preponderance have either focused exclusively on the acute polymorphic psychotic disorder (F23.0) subcategory ${ }^{22,26}$ or have examined a cohort of individuals where this subcategory was overrepresented. ${ }^{18}$ Hence, it would appear that gender ratio may be a reflection of the relative proportion of ATPD subcategories in the sampled population. It is thus not possible to draw any definite epidemiological conclusions on gender ratio in ATPD from our study because of the lack of ATPD subcategories in our data-set.

\section{Age at onset}

Our findings of an early-middle adulthood onset are in line with the previous studies of ATPD. Our mean age at onset (37.4 years) is akin to those previously reported by Jorgensen et al (37 years), Marneros et al (35.8 years) and Castagnini et al (42.2 years). ${ }^{18,19,21}$ We found a significantly higher mean age at onset in women than in men (41.8 v. 33.8 years) and a reversal of the pattern of age distribution with a higher proportion of women than men presenting after 40 years of age. Previously, Castagnini et al had found a similar later mean age at onset of ATPD in women (46.2 v. 37.8 years) and a similar reversal of the pattern of incidence rates by gender groups over 50 years. ${ }^{21}$ This latter finding is consistent with the evidence that psychotic symptoms tend to emerge later in women, perhaps owing to a protective effect exerted by oestrogens via their neuromodulatory and neuroprotective activity on the brain. ${ }^{33}$ Also our data seem to confirm that average age at onset of ATPD is higher than that of schizophrenia. ${ }^{13,30}$

\section{Diagnostic stability}

The overall diagnostic stability rate in our cohort was estimated at $53.9 \%$ over approximately 4 years, including patients presenting with recurrent (7.6\%) and mono-episodic (46.3\%) ATPD. This is similar to that previously found by Marneros et al ${ }^{19}$ (54\%), whose follow-up examinations took place on average 10.2 years after the first episode, but differs from those reported by Singh et $a l^{20}(34 \%)$ and Castagnini et $a l^{21}$ (39\%), whose observation period lasted for 3 years and 6 years respectively. Of particular note is the observation that high stability rates for ATPD, ranging from 63 to $100 \%$, have been reported in studies conducted in low- and middle-income countries. ${ }^{23,25,26}$ One interpretation of these results has been that the concept of ATPD may be applied differently to different cultural groups on account of cultural variations in the manifestation of its typical psychopathology.

Little is known about the temporal pattern of diagnostic shift in ATPD. In our research, due to the staggered entry of patients into the study we had to accommodate varying lengths of follow-up time among different patients. Kaplan-Meier analysis with censoring was used to factor in these missing data and it revealed a mean time to any diagnostic shift from first-episode ATPD of 7.6 years. Furthermore, the spectrum of observed diagnostic shifts appears to be encompassing a wide variety of psychiatric disorders as it is shown in Fig. 1. Hence, although it is possible our ATPD diagnostic stability rate is a slight overestimate as it may include misdiagnosed mental disorders or non-specific early manifestation of other psychiatric vulnerabilities, it could also be argued that changes in diagnosis after 6 or 7 years of first-episode ATPD reflect independent risks of other underlying psychiatric problems rather than an evolving disorder that first presented with ATPD. Although our data would appear to suggest that the general ICD-10 diagnostic criteria for firstepisode ATPD are of limited clinical utility, lacking as they are much in the way of predictive diagnostic specificity, it is still possible that additional criteria for specific ATPD subcategories such as the polymorphous subtype may instead reliably identify longitudinally stable clinical syndromes of benign prognosis and outcome. 


\section{Recurrence rate}

In accordance with existing data our study also revealed that 1 in $13(7.6 \%)$ of these individuals with acute and rapidly remitting psychoses will experience recurrences of similar psychotic episodes. The presence of a recurrent pattern with complete inter-episodic recovery in a proportion of patients with ATPD has been long recognised. Earlier reports found rates of a recurrent course in ATPD ranging from 10 to $50 \%^{20,21,25,34}$ but this broad range could be accounted for by differences in sampling methods and study design. Interestingly, a chronic course, which is somewhat at odds with the concept of acute transient psychoses, has also been described. ${ }^{29,35}$ Of note, Marneros et al found no significant difference in the frequency of relapses between ATPD and schizophrenia. ${ }^{13}$ These findings challenge the Kraepelinian dichotomy of functional psychoses and are more in keeping with several European psychiatric traditions as to the existence of psychotic syndromes with a favourable and relatively benign outcome. Unfortunately, the operationalised diagnostic criteria used in the current international taxonomies seem to be unable to reliably identify these individuals. In our sample neither gender nor age at first presentation appeared to be parameters significantly associated with this subgroup of patients. However, a non-significant trend for shorter length of first admission was observed.

\section{Limitations}

Although it is possible that the steady decline of psychiatric in-patient beds recorded across Scotland over the recruitment period and the potential shortening of duration of hospital admission following the introduction of home intensive treatment services in some Scottish NHS Boards may have biased the statistics on ATPD incidence rates and average length of hospital stays, it is questionable whether such changes in healthcare provision may have significantly had an impact on the clinical management of short-lived and typically florid psychoses. No information regarding deaths occurring during the follow-up period could be extracted from our data and the effect this might have had on our findings could not be estimated. Diagnostic accuracy is a common problem with administrative data since clinical diagnoses cannot be verified and validated. It is unlikely that this constitutes a major problem since uniformity of psychiatric training across Scotland and our large national cohort will have minimised the impact on the results of any diagnostic discrepancies. Moreover, the naturalistic design of the study has precluded the analysis of any confounding effect that potential therapeutic interventions may have exerted on the observed outcomes. Unfortunately, due to the nature of the data it is also impossible to exclude any previous presentation with psychotic symptoms to mental health services outside Scotland and any subsequent relapse that may have been managed in the out-patient clinic or may have lead to hospital admission outside Scotland. The lack of additional coding regarding ATPD subcategories does not permit any consideration on the clinical utility of the 'polymorphous' variety of ATPD. Also, information is missing on whether onset of symptoms was abrupt $(<48 \mathrm{~h})$ or acute ( $>48 \mathrm{~h}$ but $<2$ weeks), with the former having been reported to be associated with higher diagnostic stability. ${ }^{26}$

\section{Implications}

According to a synthesis of our and other available data, ATPD requiring hospital admission is a rare mental disorder with an incidence of about 4 per 100000 per year, which typically presents in early-middle adulthood. The gender ratio is more or less equal, although women appear to be more likely to retain their initial diagnosis of ATPD than men. As a diagnostic concept, ATPD remains inadequately validated and of limited longitudinal diagnostic stability. Around one in two people initially diagnosed with ATPD will change to a different diagnosis and one in eight will eventually develop schizophrenia. Hence, acute onset and full recovery within 1-3 months do not seem to be always associated with long-term favourable outcome. Nonetheless, almost a half of people presenting with ATPD may only have a single episode. There also appears to be a group of people who retain the diagnosis and present with recurrent episodes of illness, lending support to the view that some still poorly defined acute and rapidly remitting psychoses are not merely prodromal phases of affective or schizophrenic psychoses but a separate clinical entity, which does not fit into the traditional Kraepelinian dichotomy of functional psychoses. In this seemingly heterogeneous population of ATPD, there seems to be clinical (longer first admission to hospital) and demographic (young age at onset and male gender) variables that are associated with increased risk and earlier development of schizophrenia. Monitoring over a follow-up period of around 3-5 years may therefore be warranted in order to confirm or exclude a diagnosis of schizophrenia.

\footnotetext{
Filippo Queirazza, MD, MRCPsych, Hairmyres Hospital, East Kilbride, Glasgow; David M. Semple, MB ChB, MRCPsych, Division of Psychiatry, University of Edinburgh, Edinburgh, and Hairmyres Hospital, East Kilbride, Glasgow; Stephen M Lawrie, MD, FRCPsych, Division of Psychiatry, University of Edinburgh, UK

Correspondence: David M. Semple, Department of Mental Health, Hairmyres Hospital, Eaglesham Road, East Kilbride, Glasgow G75 8RG, UK. Email: David.Semple@lanarkshire.scot.nhs.uk

First received 21 Feb 2013, final revision 8 Jul 2013, accepted 25 Oct 2013
}

\section{References}

1 Marneros A. Beyond the Kraepelinian dichotomy: acute and transient psychotic disorders and the necessity for clinical differentiation. Br J Psychiatry 2006; 189: 1-2.

2 Kraepelin E. Psychiatrie [Psychiatry]. Barth, 1896.

3 Pillmann F, Marneros A. Brief and acute psychoses: the development of concepts. Hist Psychiatry 2003; 14: 161-77.

4 Kleist K. Uber zykloide und epileptoide psychosen und uber die frgae der degenerationpsychosen [Cycloid, paranoid and epileptoid psychoses and the problem of degenerative psychoses]. Schweiz Arch Neurol Neurochir Psychiatr 1928; 23: 3-37.

5 Leonhard K. Aufteilung der Endogenen Psychosen [Classification of the Endogenous Psychoses]. Akademie, 1957.

6 Magnan V. Lecon Cliniques sur les Maladies Mentales [Clinical Lessons on Mental Illnesses]. Bataille, 1893.

7 Ey $\mathrm{H}$. Les bouffees delirantes et les psychoses hallucinatoires aigues ['Bouffées délirantes' and acute hallucinatory psychoses]. Etude n. 23. In Etudes psychiatriques: 203-324. Desclee de Brouwer, 1954.

8 Wimmer A (ed.) Psykogene Sindssygdomsformer [Psychogenic psychoses]. In Sct Hans Hospital 1816-1915 Jubilaeumsskrifts: 85-216. Gad, 1916.

9 Langfeldt G. The Schizophreniform States. Munksgaard, 1939.

10 World Health Organization. The ICD-10 Classification of Mental and Behavioural Disorders. Clinical Descriptions and Diagnostic Guidelines. WHO, 1992.

11 Pillmann F, Haring A, Balzuweit S, Bloink R, Marneros A. Concordance of acute and transient psychoses and cycloid psychoses. Psychopathology 2001; 34: 305-11.

12 Pillmann F, Haring A, Balzuweit S, Bloink R, Marneros A. Bouffee delirante and ICD-10 acute and transient psychoses: a comparative study. Aust N Z J Psychiatry 2003; 37: 327-33.

13 Marneros A, Pillmann F, Haring A, Balzuweit S, Bloink R. What is schizophrenic in acute and transient psychotic disorder? Schizophr Bull 2003; 29: 311-23. 
14 Jager M, Bottlender R, Strauss A, Moller HJ. On the descriptive validity of ICD-10 schizophrenia: empirical analyses in the spectrum of non-affective functional psychoses. Psychopathology 2003; 36: 152-9.

15 Pillmann $\mathrm{F}$, Marneros A. Longitudinal follow-up in acute and transient psychotic disorders and schizophrenia. Br J Psychiatry 2005; 187: 286-7.

16 Robins E, Guze SB. Establishment of diagnostic validity in psychiatric illness: its application to schizophrenia. Am J Psychiatry 1970; 126: 983-7.

17 Kendell RE. Clinical validity. Psychol Med 1989; 19: 45-55.

18 Jorgensen $\mathrm{P}$, Bennedsen B, Christensen J, Hyllested A. Acute and transient psychotic disorder: a 1-year follow-up study. Acta Psychiatr Scand 1997; 96 150-4.

19 Marneros A, Pillmann F, Haring A, Balzuweit S, Bloink R. Features of acute and transient psychotic disorders. Eur Arch Psychiatry Clin Neurosci 2003; 253: $167-74$.

20 Singh SP, Burns T, Amin S, Jones PB, Harrison G. Acute and transient psychotic disorders: precursors, epidemiology, course and outcome. Br J Psychiatry 2004; 185: 452-9.

21 Castagnini A, Bertelsen A, Berrios GE. Incidence and diagnostic stability of ICD-10 acute and transient psychotic disorders. Compr Psychiatry 2008; 49 255-61.

22 Suda K, Hayashi N, Hiraga M. Predicting features of later development of schizophrenia among patients with acute and transient psychotic disorder Psychiatry Clin Neurosci 2005; 59: 146-50.

23 Amini H, Alaghband-rad J, Omid A, Sharifi V, Davari-Ashtiani R, Momeni F, et al. Diagnostic stability in patients with first-episode psychosis. Australas Psychiatry 2005; 13: 388-92.

24 Abe T, Otsuka K, Kato S. Long-term clinical course of patients with acute polymorphic psychotic disorder without symptoms of schizophrenia. Psychiatry Clin Neurosci 2006; 60: 452-7.

25 Thangadurai P, Gopalakrishnan R, Kurian S, Jacob KS. Diagnostic stability and status of acute and transient psychotic disorders. Br J Psychiatry 2006; 188 293.
26 Sajith SG, Chandrasekaran R, Sadanandan Unni KE, Sahai A. Acute polymorphic psychotic disorder: diagnostic stability over 3 years. Acta Psychiatr Scand 2002; 105: 104-9.

27 Okasha A, el Dawla AS, Khalil AH, Saad A. Presentation of acute psychosis in an Egyptian sample: a transcultural comparison. Compr Psychiatry 1993; 34: 4-9.

28 Chang WC, Pang SL, Chung DW, Chan SS. Five-year stability of ICD-10 diagnoses among Chinese patients presented with first-episode psychosis in Hong Kong. Schizophr Res 2009; 115: 351-7.

29 Moller HJ, Jager M, Riedel M, Obermeier M, Strauss A, Bottlender R. The Munich 15-year follow-up study (MUFUSSAD) on first-hospitalized patients with schizophrenic or affective disorders: assessing courses, types and time stability of diagnostic classification. Eur Psychiatry 2011; 26: 231-43.

30 Salvatore $\mathrm{P}$, Baldessarini RJ, Tohen M, Khalsa HM, Sanchez-Toledo JP, Zarate Jr CA, et al. McLean-Harvard international first-episode project: two-year stability of ICD-10 diagnoses in 500 first-episode psychotic disorder patients. J Clin Psychiatry 2011; 72: 183-93.

31 Gaebel W, Zielasek J, Cleveland HR. Classifying psychosis - challenges and opportunities. Int Rev Psychiatry 2012; 24: 538-48.

32 Marneros A, Pillmann F, Haring A, Balzuweit S, Bloink R. Is the psychopathology of acute and transient psychotic disorder different from schizophrenic and schizoaffective disorders? Eur Psychiatry 2005; 20: $315-20$.

33 Boerma MA, van der Stel JC, van Amelsvoort T, Linszen DH, de Haan L. Women, schizophrenia and oestrogen; neurobiological hypotheses and hormonetherapy studies [in Dutch]. Tijdschr Psychiatr 2010; 52: 235-44.

34 Jager MDM, Hintermayr M, Bottlender R, Strauss A, Moller HJ. Course and outcome of first-admitted patients with acute and transient psychotic disorders (ICD-10: F23). Focus on relapses and social adjustment. Eur Arch Psychiatry Clin Neurosci 2003; 253: 209-15.

35 Jager M, Bottlender R, Strauss A, Moller HJ. The classification of functional psychoses: the impact of ICD-10 diagnoses (research diagnostic criteria) for the prediction of the long-term course [in German]. Fortschr Neurol Psychiatr 2004; 72: 70-8.

\section{0 woos}

\section{Jealousy}

Gillian Rathbone

Jealousy is triangular unlike envy and greed. Its target is the perceived rival for the affection of a third, the good object, whom he fears to lose. Freud describes the earliest unconscious jealousy as from the child against his father for exclusive rights to his mother. Successful resolution of the Oedipus complex requires the child to accept defeat and underpins development of capacities for concern and reparation, internalisation of parental values, and the beginning of separation from the mother. Jealousy forms a spectrum between persecution and healthy competition, dependent on degrees of envy and admiration, and ultimately, may promote emulation. 